University of Nebraska - Lincoln

DigitalCommons@University of Nebraska - Lincoln

8-1-1975

\title{
Effect of Elliptically Polarized Light on the Angular Distribution of Photoelectrons
}

James A. R. Samson

University of Nebraska - Lincoln

Anthony F. Starace

University of Nebraska-Lincoln, astarace1@unl.edu

Follow this and additional works at: https://digitalcommons.unl.edu/physicsstarace

Part of the Physics Commons

Samson, James A. R. and Starace, Anthony F., "Effect of Elliptically Polarized Light on the Angular Distribution of Photoelectrons" (1975). Anthony F. Starace Publications. 155.

https://digitalcommons.unl.edu/physicsstarace/155

This Article is brought to you for free and open access by the Research Papers in Physics and Astronomy at DigitalCommons@University of Nebraska - Lincoln. It has been accepted for inclusion in Anthony F. Starace Publications by an authorized administrator of DigitalCommons@University of Nebraska - Lincoln. 
Published in Journal of Physics B: Atomic and Molecular Physics 8:11 (August 1, 1975), pp.

1806-1809. Copyright $\odot 1975$ Institute of Physics Publishing. Used by permission. http:// www.iop.org/EJ/journal/JPhysB

Submitted January 27, 1975; revised April 2, 1975.

\title{
Effect of Elliptically Polarized Light on the Angular Distribution of Photoelectrons
}

\author{
James A. R. Samson and Anthony F. Starace \\ Behlen Laboratory of Physics, University of Nebraska-Lincoln \\ Lincoln, Nebraska 68508, USA
}

\begin{abstract}
The angular distribution of photoelectrons predicted for elliptically polarized light is shown to be the same as that predicted for partially polarized light having incoherent perpendicular electric field components equal to the electric field components along the major and minor axes of the ellipse.
\end{abstract}

Increasing use by atomic experimenters of synchrotron radiation, which is known to be elliptically polarized (Sokolov and Ternov 1957, Joos 1960) and of metallic mirrors, which cause reflected light to be elliptically polarized (Jenkins and White 1957), has focused attention on the expected angular distribution of photoelectrons produced by elliptically polarized light. Schmidt (1973) has obtained an expression for the angular distribution of photoelectrons produced by elliptically polarized light that appears quite different from the expression obtained by Samson $(1969,1970)$ for photoelectrons produced by partially polarized light. We show here, however, that Schmidt's formula reduces to that of Samson when one considers the electric vector components along the major and minor axes of the ellipse that characterizes the elliptically polarized beam as the two perpendicular, incoherent electric vector components of a partially polarized beam.

We consider light incident along the $z$ axis interacting with an unpolarized atom at the origin via the electric dipole interaction and ejecting a photoelectron along a direction described by the angles $\theta_{x^{\prime}} \theta_{y^{\prime}}$ and $\theta_{z}$ (cf Figure 1). If the incident light is linearly polarized, then the angular distribution of photoelectrons produced in many such collisions is described by the differential cross section (Yang 1948, Cooper and Zare 1969):

$$
\frac{\mathrm{d} \sigma}{\mathrm{d} \Omega}=\frac{\sigma}{4 \pi}\left[1+\beta P_{2}(\cos \theta)\right] .
$$

In equation (1), $\sigma$ is the total cross section, $\beta$ is the asymmetry parameter,

$$
P_{2}(\cos \theta) \equiv 3 / 2 \cos ^{2} \theta-1 / 2,
$$

and $\theta$ is measured from the electric vector of the incident light.

Now by "partially polarized light," we mean partially linearly polarized light because partially circularly polarized light is equivalent to unpolarized light plus circularly polarized light, and circularly polarized light gives the same photoelectron angular distribution as unpolarized light (Peshkin 1970, Jacobs 1972). Thus we may for our present purpose consider partially polarized light as equivalent to two incoherent linearly polarized beams vibrating along orthogonal axes (Born and Wolf 1959, § 10.8.2). 


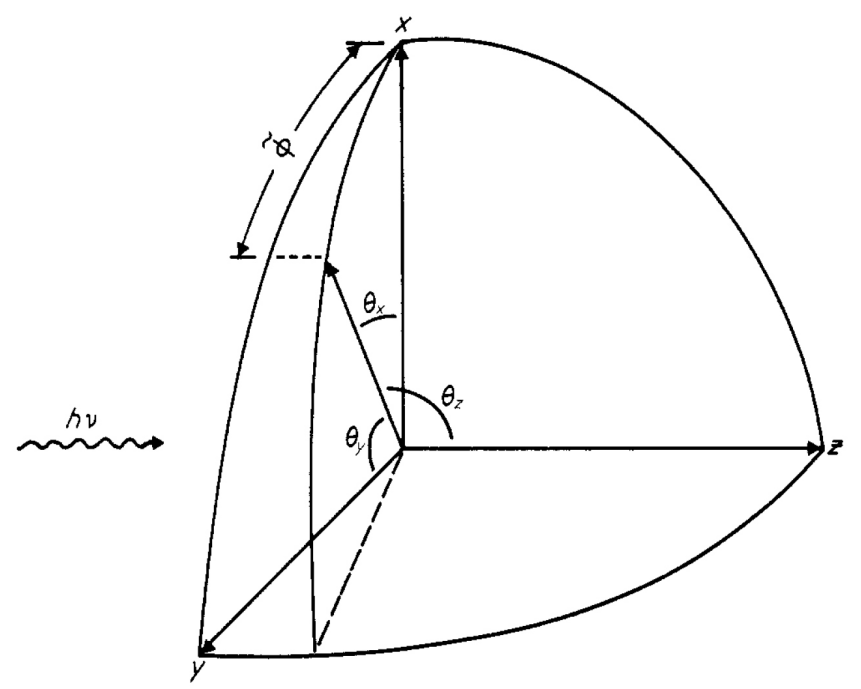

Figure 1. Orientation of the photoelectron with respect to the $x, y$, and $z$ axes. The photon $h v$ is incident along the $z$ axis. The azimuthal angle of the photoelectron with respect to the $x$ axis is indicated by $\tilde{\phi}$.

The resulting angular distribution is thus obtained as the sum of two differential cross sections having the form of equation (1) :

$$
\frac{\mathrm{d} \sigma}{\mathrm{d} \Omega}=\frac{I_{x}}{I_{0}} \frac{\sigma}{4 \pi}\left[1+\beta P_{2}\left(\cos \theta_{x}\right)\right]+\frac{I_{y}}{I_{0}} \frac{\sigma}{4 \pi}\left[1+\beta P_{2}\left(\cos \theta_{y}\right)\right] .
$$

In equation (2) we have weighted the superposition by the coefficients $\left(I_{x} / I_{0}\right)$ and $\left(I_{y} / I_{0}\right)$ describing the fraction of light intensity along the $x$ and $y$ axes, where $I_{0}=$ $I_{x}+I_{y}$. Note that equation (2) above is identical to equation (5) of Samson (1969) upon making the substitutions

$$
A=\frac{1}{I_{0}} \frac{\sigma}{4 \pi}\left(1-\frac{\beta}{2}\right)
$$

and

$$
B=\frac{1}{I_{0}} \frac{\sigma}{4 \pi} \frac{3}{2} \beta,
$$

and also making the appropriate changes in the labels of the coordinate axes.

Defining the polarization of the incident light beam as

$$
p \equiv \frac{I_{y}-I_{x}}{I_{x}+I_{y}}
$$

and using the geometric relation,

$$
\cos ^{2} \theta_{x}+\cos ^{2} \theta_{y}+\cos ^{2} \theta_{z}=1
$$

we may rewrite equation (2) as :

$$
\frac{\mathrm{d} \sigma}{\mathrm{d} \Omega}=\frac{\sigma}{4 \pi}\left\{1-\frac{1}{2} \beta\left[P_{2}\left(\cos \theta_{z}\right)-\frac{3}{2} p\left(\cos ^{2} \theta_{x}-\cos ^{2} \theta_{y}\right)\right]\right\} .
$$


Equation (7) is to be compared with equation (3) of Schmidt (1973) for the differential cross section appropriate for elliptically polarized incident light, namely

$$
\frac{\mathrm{d} \sigma}{\mathrm{d} \Omega}=\frac{\sigma}{4 \pi}\left\{1-\frac{1}{2} \beta\left[P_{2}\left(\cos \theta_{z}\right)-\frac{3}{2} A \sin ^{2} \theta_{z}\right]\right\}
$$

In equation (8) Schmidt defines $A$ as

$$
A \equiv \cos 2 \omega \cos 2 \tilde{\phi}
$$

where

$$
\tan \omega=E_{b} / E_{a}
$$

and $\tilde{\phi}$ is defined in our figure $1 . E_{b}$ and $E_{a}$ are the components of the electric vector along the major and minor axes of the ellipse.

It can easily be shown now that equation (8) and equation (7) are identical provided one sets $E_{b}=E_{x}$ and $E_{a}=E_{y}$. That is, provided one orients our coordinate axes in Figure 1 along the major and minor axes of the ellipse. For then

$$
\cos 2 \omega=\cos ^{2} \omega-\sin ^{2} \omega=\frac{E_{y}^{2}-E_{x}^{2}}{E_{x}^{2}+E_{y}^{2}}=\frac{I_{y}-I_{x}}{I_{x}+I_{y}}=p
$$

and from Figure 1,

$$
\cos 2 \tilde{\phi}=1-2 \sin ^{2} \tilde{\phi}=1-2 \frac{\cos ^{2} \theta_{y}}{\sin ^{2} \theta_{z}} .
$$

Combining equations (6), (9), (1 1), and (12) we obtain,

$$
A \sin ^{2} \theta_{z}=p\left(\cos ^{2} \theta_{x}-\cos ^{2} \theta_{y}\right)
$$

which proves that equation (8) and equation (7) are identical.

We conclude that, fortunately for atomic experimenters, elliptically polarized light and partially polarized light give identical results for the angular distribution of photoelectrons. The general formula for partially polarized light, equation (7), may be used for elliptically polarized light, provided one knows the orientation of the major and minor axes of the ellipse and the ratio of the electric vector magnitudes along these axes. This equivalence-that we have demonstrated algebraically - may be understood qualitatively as follows: from the Stokes parameter characterization of a light beam, elliptically polarized light differs from partially linearly polarized light in that the former has a non-zero $s_{3}$, where $s_{3}$ measures the presence of circular polarization (Born and Wolf 1959, § 10.8.3). This component of circular polarization, which leads also to the phase coherence (measured by the Stokes parameters $s_{2}$ and $s_{3}$ ) of the elliptically polarized beam (Born and Wolf 1959, § 10.8.3), does not however affect the photoelectron angular distribution (Peshkin 1970, Jacobs 1972). Hence, as far as the angular distribution of photoelectrons is concerned, it makes no difference whether one uses elliptically polarized or partially linearly polarized light. However, these different light polarizations will affect other measurable properties of the final state that are sensitive to the presence of circularly polarized light, such as the photoelectron spin polarization (Jacobs 1972, p. 2266). 


\section{Acknowledgments}

We are grateful to Professors U. Fano and J. Macek for the interpretation of our result in terms of Stokes parameters. We also wish to thank Professor D. Dill and Dr. V. Schmidt for helpful comments on our manuscript. This work was supported by the National Aeronautics and Space Administration under Grant NGR 28-004-021.

\section{References}

Born, M., and Wolf, E. 1959 Principles of optics (New York: Pergamon)

Cooper, J., and Zare, R. N. 1969 Lectures in Theoretical Physics, vol. 11c, ed. S. Geltman, K. T. Mahanthappa, and W. E. Britten (New York: Gordon and Breach), pp. 317-37

Jacobs, V. L. 1972 J. Phys. B: Atom. Molec. Phys. 5: 2257-71

Jenkins, F. A., and White, H. E. 1957 Fundamentals of Optics 3rd ed. (New York: McGraw-Hill)

Joos, P. 1960 Phys. Rev. Lett. 4 :558-9

Peshkin, M. 1970 Advances in Chemical Physics vol. 18 (New York: Interscience) pp 1-14

Samson, J. A. R. 1969 J. Opt. Soc. Am. 59: 356-7

1970 Phil. Trans. R. Soc. A 268: 141-6

Schmidt, V. 1973 Phys. Lett. 45A: 634

Sokolov, A. A., and Ternov, I. M. 1957 Sov. Phys.JJETP 4: 396-400

Yang, C. N. 1948 Phys. Rev. 74: 76472

See Corrigendum (1979), following. 
Published in Journal of Physics B: Atomic and Molecular Physics, Vol. 12, No. 23, (Dec. 14, 1979), p. 3993.

Copyright () 1979 The Institute of Physics. Used by permission.

\section{Corrigendum to}

\section{Effect of elliptically polarised light on the angular distribution of photoelectrons}

J. A. R. Samson and A. F. Starace, 1975, J. Phys. B: Atom. Molec. Phys. 8 1806-9

A sign error in the definition of the polarisation $p$ in equation (5) necessitates the following corrections.

(i) Equation (5) should read

$$
p \equiv \frac{I_{x}-I_{y}}{I_{x}+I_{y}}
$$

(ii) Two lines above equation (11) we make the associations, $E_{b}=E_{y}$ and $E_{a}=E_{x}$.

(iii) Equation (11) requires $x$ and $y$ subscripts to be interchanged.

The substance and conclusions of the papers are unchanged.

We wish to thank Dr Keh-Ning Huang for pointing out this error. 